\title{
Spontaneous Periodic Deformations in Nonchiral Planar-Aligned Bimesogens with a Nematic-Nematic Transition and a Negative Elastic Constant
}

\author{
V. P. Panov \\ Trinity College Dublin, Ireland \\ J. K. Vij \\ Trinity College Dublin, Ireland, jvjj@tcd.ie \\ Yuri Panarin \\ Technological University Dublin, yuri.panarin@tudublin.ie
}

See next page for additional authors

Follow this and additional works at: https://arrow.tudublin.ie/engscheleart2

Part of the Electrical and Computer Engineering Commons

\section{Recommended Citation}

Kohlmeier, A. et al. (2010). Spontaneous periodic deformations in nonchiral planar-aligned bimesogens with a nematic-nematic transition and a negative elastic constant. Physical Review Letters, vol. 105, pp. 1-4. DOI:10.1103/PhysRevLett.105.167801

This Article is brought to you for free and open access by the School of Electrical and Electronic Engineering at ARROW@TU Dublin. It has been accepted for inclusion in Articles by an authorized administrator of ARROW@TU Dublin. For more information, please contact arrow.admin@tudublin.ie, aisling.coyne@tudublin.ie, gerard.connolly@tudublin.ie.

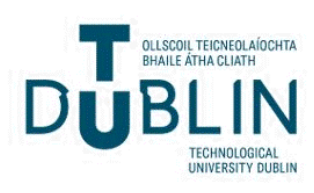




\section{Authors}

V. P. Panov, J. K. Vij, Yuri Panarin, A. Kohlmeier, M. G. Tamba, R. A. Lewis, and G. H. Mehl

This article is available at ARROW@TU Dublin: https://arrow.tudublin.ie/engscheleart2/164 


\title{
Spontaneous Periodic Deformations in Nonchiral Planar-Aligned Bimesogens with a Nematic-Nematic Transition and a Negative Elastic Constant
}

\author{
V.P. Panov, ${ }^{1}$ M. Nagaraj, ${ }^{1}$ J. K. Vij, ${ }^{1, *}$ Yu. P. Panarin, ${ }^{1}$ A. Kohlmeier, ${ }^{2}$ M. G. Tamba, ${ }^{2}$ R. A. Lewis,${ }^{2}$ and G. H. Mehl ${ }^{2}$ \\ ${ }^{1}$ Department of Electronic and Electrical Engineering, Trinity College, University of Dublin, Dublin 2, Ireland \\ ${ }^{2}$ Department of Chemistry, University of Hull, HU6 7RX, United Kingdom
}

(Received 17 June 2010; published 12 October 2010)

\begin{abstract}
Hydrocarbon linked mesogenic dimers are found to exhibit an additional nematic phase below the conventional uniaxial nematic phase as confirmed by x-ray diffraction. The phase produces unusual periodic stripe domains in planar cells. The stripes are found to be parallel to the rubbing direction (in rubbed cells) with a well-defined period equal to double the cell gap. The stripes appear without external electromagnetic field, temperature or thickness gradients, rubbing or hybrid alignment treatments. Simple modeling proposes a negative sign for at least one of the two elastic constants: splay and twist, as a necessary condition for the observed pattern.
\end{abstract}

DOI: 10.1103/PhysRevLett.105.167801

PACS numbers: 61.30.Eb, 61.30.Gd

A variety of stable periodic deformations in nematic liquid crystal (NLC) samples were observed and analyzed some time ago [1]. These attract attention due to potential applications of variable grating mode (VGM) optical computing devices [2] as well as for convenient measurements of the intrinsic physical properties of liquid crystals [1]. Periodic stripe patterns easily observed by polarizing optical microscopy (POM) are normally caused by the presence of a helical structure [1] or by the application of external fields: magnetic [3], electric [4], or mechanical stress $[5,6]$. In planar geometry of the cell, the spontaneous deformations of nematic director have also been proposed theoretically [7-10].

In this work we report the observation of at least an additional liquid crystalline phase in the temperature range below the conventional uniaxial nematic $\left(N_{u}\right)$ found in three materials of hydrocarbon linked mesogenic dimers (Fig. 1). The presence of spontaneous deformation patterns in planar cells is one of the distinct features of this phase (designated as $N_{x}$ ). We also provide a simple theoretical analysis for explaining the existence of the patterns and their difference from the observed or predicted stripes that have been reported in the literature. Despite the obvious chemical differences, different phase transitions temperatures (as determined by POM), and even different signs of dielectric anisotropy (positive for $M 1$ and negative for $M 2$, M3), the three studied materials (Fig. 1) exhibit two nematic phases with a characteristic striped pattern appearing a few degrees below the $N_{u}-N_{x}$ transition in planar cells, suggesting a characteristic feature of the self-assembly of molecules. POM and differential scanning calorimetry show a clear difference in between the two phases with a first order $N_{u}-N_{x}$ phase transition $(\Delta H=0.14 \mathrm{~kJ} / \mathrm{mol})$ for $M 1$. For other materials the enthalpy associated with the $N_{u}-N_{x}$ transition is very small $(\Delta H \leq 0.01 \mathrm{~kJ} / \mathrm{mol})$, albeit for all of the materials the change in the optical textures is obvious, sharp, and occurs at a well-defined temperature.
Figures 2(a)-2(c) present POM microphotographs obtained for $M 2$ at different temperatures in a $7 \mu \mathrm{m}$ thick planar unrubbed cell. At higher temperature [Fig. 2(a)] the Schlieren texture corresponds to that for the conventional nematic, Fig. 2(b) shows the $N_{x}$ phase just below the transition from the $N_{u}$. The pattern in the $N_{x}$ phase exhibits a number of fine defects and does not appear to be as uniform as that of $N_{u}$ and the thermal fluctuations characteristic of the classical nematic phase are practically invisible in the $N_{x}$. Figure 2(c) shows the $N_{x}$ phase at a lower temperature when the stripe pattern appears. In a homeotropically aligned cell both the $N_{x}$ and $N_{u}$ phases appear dark under crossed polarizers. The $N_{x}$ phase can be distinguished by the absence of the thermal fluctuations. On further cooling below the $N_{u}-N_{x}$ phase transition the texture remains the same throughout the entire range of the $N_{x}$ phase. No optical rotation or segregation into domains is observed in homeotropic cells. The stripe patterns appear on cooling and they gradually fill the entire area of the cell. However, once having established, there is no significant change with temperature. Application of a small electric field (up to $1 \mathrm{~V} / \mu \mathrm{m}$ ) along the cell normal does not affect the observed pattern irrespective of the sign of the dielectric anisotropy of the material. The possibility that this phase could be ferroelectric nematic is ruled out by an absence of (i) a detectable switching current as per our experiments and of (ii) the fundamental harmonic of the

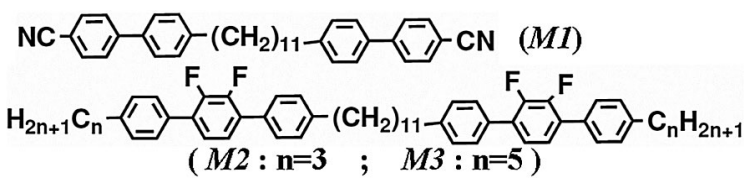

FIG. 1. Liquid crystalline materials used. $M 1: \mathrm{Cr}\left(91^{\circ} \mathrm{C}\right) N_{x}$ $\left(109^{\circ} \mathrm{C}\right) N_{u}\left(127^{\circ} \mathrm{C}\right)$ Iso; $M 2$ : $\mathrm{Cr}\left(95^{\circ} \mathrm{C}\right) N_{x}\left(137^{\circ} \mathrm{C}\right) N_{u}$ $\left(193{ }^{\circ} \mathrm{C}\right)$ Iso; $M 3: \mathrm{Cr}\left(85^{\circ} \mathrm{C}\right) N_{x}\left(123^{\circ} \mathrm{C}\right) N_{u}\left(170^{\circ} \mathrm{C}\right)$ Iso. $N_{x}$ is the unusual nematic phase under investigation. 

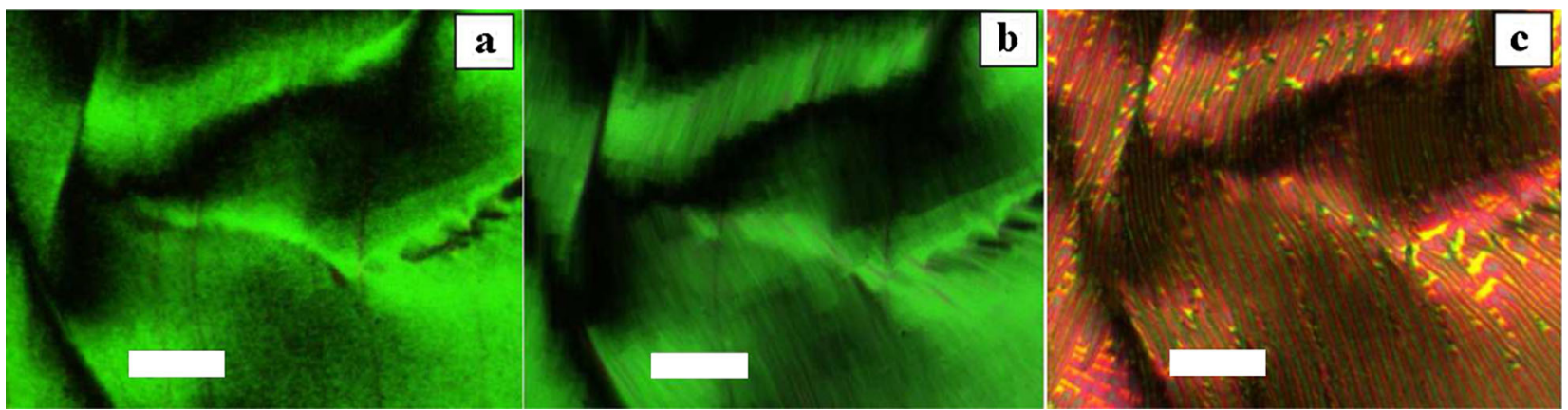

FIG. 2 (color online). A $7 \mu \mathrm{m}$ planar unrubbed cell of $M 2$ between crossed polarizers at different temperatures. The length of the white bar is $100 \mu \mathrm{m}$ (a) $N_{u}$ phase, $T=140^{\circ} \mathrm{C}$, (b) $N_{x}$ phase, uniform, $T=135^{\circ} \mathrm{C}$, (c) $N_{x}$ phase, striped, $T=120^{\circ} \mathrm{C}$.

transmission signal when an ac electric field is applied across the cell [11]. Hence ferroelectricity does not cause the observed periodic deformation.

In (anti)parallel rubbed planar cells the stripes are highly uniform and their period $D$ is found to be equal to double the cell gap $d$ within the experimental error [Fig. 3(a)] when the cell thickness is varied from $2 \mu \mathrm{m}$ (the lowest cell gap we have experimented with) to about $10 \mu \mathrm{m}$ (for thicker cells the stripes break into a two-dimensional pattern). The color of the pattern varies from gray in thin cells to multicolor stripes in thicker cells. A large number of higher order interference colors [Fig. 3(a), bottom inset] in thicker cells leads us to conclude that there is a gradual
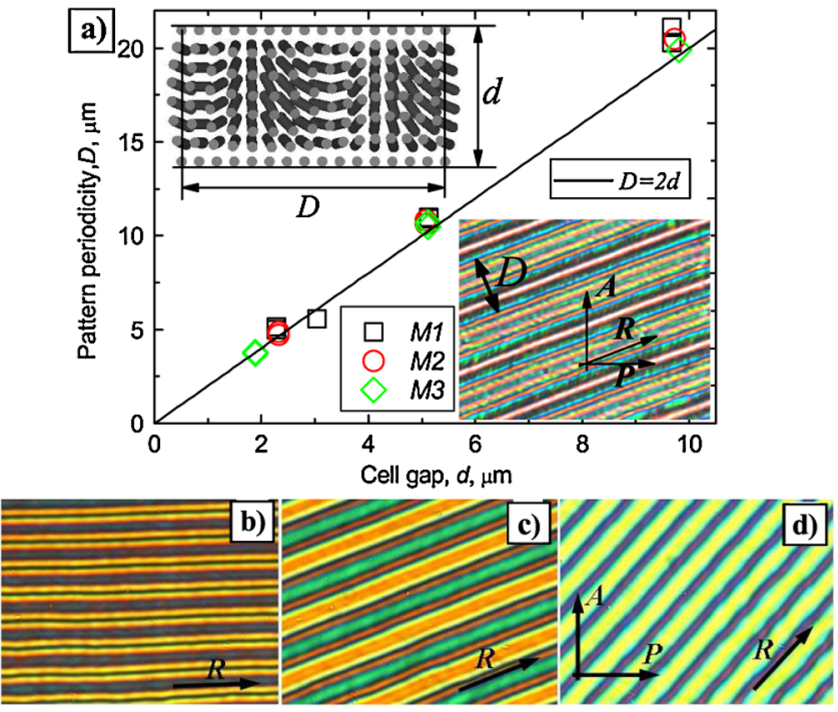

FIG. 3 (color online). (a) Pattern periodicity in different materials as a function of the cell gap. $D=2 d$ is an eye-guide line. The insets explain the definition of the pattern period $D$ using (bottom) POM image of $M 3$ at $110^{\circ} \mathrm{C}$ in $9 \mu \mathrm{m}$ commercial cell with the rubbing direction rotated by $20^{\circ}$ from the polarizer axis and (top) a schematic cross section of the proposed molecular distribution by the $y-z$ plane. (b)-(d) Rotation of a $5 \mu \mathrm{m}$ planar rubbed cell in crossed polarizers. $M 3, T=110^{\circ} \mathrm{C}$. Image height $\sim 40 \mu \mathrm{m}$. The angle between the polarizer $(\mathbf{P})$ and the rubbing direction $(\mathbf{R})$ is (b) $0^{\circ}$, (c) $20^{\circ}$, (d) $45^{\circ}$. periodic change in integrated birefringence producing a wide range of retardation values. This is true for both commercial (EHC. Co., KSRP-XX-A2 \| P1NSS) and homemade cells (aligning agent RN1175, Nissan Chemicals, Japan). The cell gaps were measured using a spectrometer and the pattern periodicity is obtained by a microscope-attached CCD camera calibrated with an object micrometer. These provide measurement errors negligible compared to the unavoidable nonuniformities of the pattern and cell gaps.

When a cell is rotated between the crossed polarizers [Figs. 3(b)-3(d)] the color pattern changes as expected from a space-modulated retarder. From the analysis of the intensity and the colors appearing between the crossed polarizers, we suggest that both the birefringence and its axis deviation from the rubbing direction (in the $x-y$ plane, see Fig. 4) are periodic functions of the $y$ coordinate. Moreover, the maxima of the birefringence correspond to the extreme deviations of its axis from the rubbing direction. When the projection of the director to the $x-y$ plane coincides with the rubbing direction, the birefringence reaches its minimum value. Here we assume that the birefringence is integrated over the cell thickness along

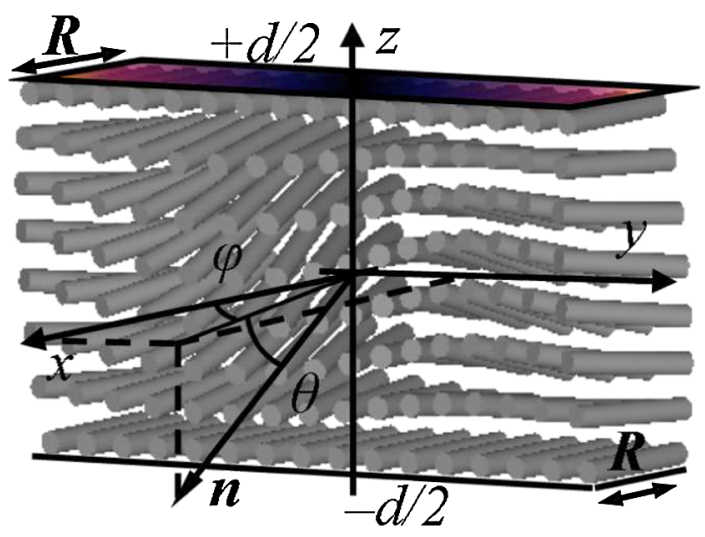

FIG. 4 (color online). Definition of the coordinates and angles of the director distribution. Rubbing direction $(\mathbf{R})$ is parallel to the $x$ axis. $\theta$ and $\varphi$ are the polar and the azimuthal angles, respectively. 


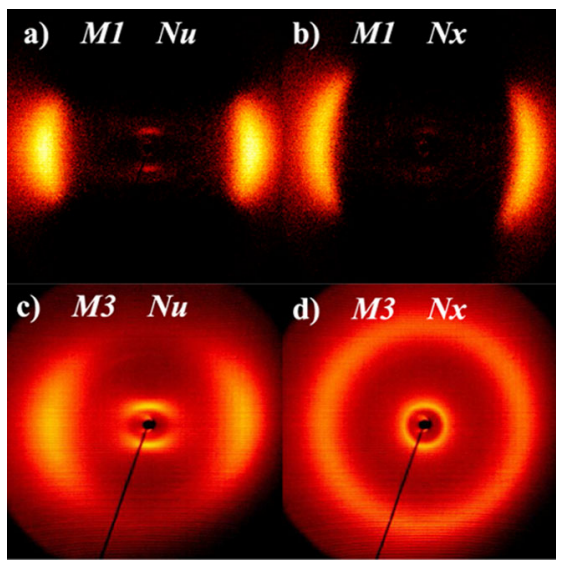

FIG. 5 (color online). XRD patterns of samples aligned by $\sim 1 \mathrm{~T}$ magnetic field on cooling. (a),(b) $M 1$, (c),(d) M3, (a), (c) $N_{u}$ phase, (b),(d) $N_{x}$ phase.

the $z$ axis and its change is caused by the change in the polar angle $\theta$ (Fig. 4). Therefore maximum values of the apparent birefringence at a particular value of the $y$ coordinate imply $\theta=0$ across most of the cell thickness at the point. A detailed investigation is restricted by the resolution limit of the optical microscope; moreover, the 2D image cannot carry the information about the molecular distribution along the $z$ axis. However, we estimate that the maximum deviation of the birefringence axis from the rubbing direction is at least $\pm 15^{\circ}$ and the variation between the maximum and minimum values of birefringence is at least $20 \%$.

Figures 5(a) and 5(b) show x-ray diffractograms of $M 1$ at $120^{\circ} \mathrm{C}\left(N_{u}\right.$ phase $)$ and $100{ }^{\circ} \mathrm{C}\left(N_{x}\right.$ phase $)$, respectively. For clarity the intensity recorded in the isotropic phase $\left(130{ }^{\circ} \mathrm{C}\right)$ has been subtracted from both patterns. The diffractograms show typical features of a nematic phase: a diffuse wide angle scattering located around the equator, dominating the x-ray diffraction (XRD) pattern and a diffuselike scattering on the meridian in the small angle region. The position, and the intensity of the small angle scattering change only slightly going from the $N_{u}$ to the $N_{x}$ phase. For $M 3$ a broadly similar behavior is detected. Typical diffractograms in the $N_{u}\left(160{ }^{\circ} \mathrm{C}\right)$ and $N_{x}$ $\left(100^{\circ} \mathrm{C}\right)$ phases are shown in Figs. $5(\mathrm{c})$ and 5(d), respectively. No significant increase in the small angle intensity occurs on transition into the low temperature $N_{x}$ phase in all the three materials. This confirms the absence of the layer formation and excludes a smectic phase.

Let us consider the simplest nematic director field as $\mathbf{n}=(\cos \theta \cos \varphi, \cos \theta \sin \varphi, \sin \theta)$ satisfying the above conditions. Here $d$ is the cell gap; $\varphi$ and $\theta$ are the azimuthal and polar angles of deviation from the uniform planar state; $x, y$, and $z$ are the Cartesian coordinates defined in Fig. 4.

$$
\varphi=\Phi \cos (\pi z / d) \sin (\pi y / d)
$$

$$
\theta=\Theta \cos (\pi z / d) \cos (\pi y / d) .
$$

While this director distribution at the cell surfaces $(z=$ $\pm d / 2$ ) corresponds to the ideal planar alignment along the $x$ axis, in the bulk $(z=0)$ extremes of the polar angle (i.e., minima of apparent birefringence) alternate with extremes of the azimuthal angle as found in the experiment. Since the experimental data show the same linear dependence between the cell gap and the pattern periodicity for all the materials and alignment layers, we do not need to introduce an anchoring energy or extrapolation length in our model. Then the classical form of the elastic energy density per unit of the $x$ axis is given as

$$
\begin{aligned}
F= & \int_{-d / 2}^{d / 2} \int_{-d}^{d}\left\{K_{11}(\nabla \cdot \mathbf{n})^{2}+K_{22}[\mathbf{n} \cdot(\nabla \times \mathbf{n})]^{2}\right. \\
& \left.+K_{33}[\mathbf{n} \times(\nabla \times \mathbf{n})]^{2}\right\} d y d z .
\end{aligned}
$$

Here $K_{11}, K_{22}$, and $K_{33}$ are the splay, twist, and bend elastic constants, respectively. The integration limits correspond to the stripe domain period of the $y$ axis and the cell surfaces for the $z$ axis. The divergence terms such as $K_{24}$ and $K_{13}$ do not appear in the final result as their influence in this symmetric geometry vanishes on integration.

In order to simplify the analysis we assume that the structure modulation amplitude is the same for both azimuthal and polar angles (i.e., $\Phi=\Theta$ ) and is small enough for the director field components to be truncated at $\Theta^{4}$ in the Taylor series expansion. Then the free energy can be obtained as

$$
\begin{aligned}
F= & \left(\pi^{2} K_{11}+\pi^{2} K_{22}\right) \Theta^{2} \\
& +\left(-\frac{9 \pi^{2}}{32} K_{11}-\frac{17 \pi^{2}}{32} K_{22}+\frac{\pi^{2}}{2} K_{33}\right) \Theta^{4}+O\left(\Theta^{6}\right) .
\end{aligned}
$$

Differentiating Eq. (4) over $\Theta$ gives a third degree polynomial which apart from the trivial solution $\Theta_{1}=0$ corresponding to the uniform structure has two roots that correspond potentially to the spontaneous periodic distortion in the liquid crystalline system.

$$
\Theta_{2,3}= \pm 4 \frac{\sqrt{\left(9 K_{11}+17 K_{22}-16 K_{33}\right)\left(K_{11}+K_{22}\right)}}{9 K_{11}+17 K_{22}-16 K_{33}} .
$$

For the distortion to appear at least two conditions must be satisfied: (a) $\Theta_{2,3}$ have to be real and (b) the free energy at $\Theta_{2,3}$ must be less than the one for the uniform structure $F\left(\Theta_{1}\right)$. On substituting (5) into (4) and comparing with $F\left(\Theta_{1}=0\right)=0$, we obtain the energy condition

$$
\left(9 K_{11}+17 K_{22}-16 K_{33}\right)<0 .
$$

Considering the value under the root in Eq. (5) to be positive in the context of Eq. (6) gives the real root existence condition: 


$$
\left(K_{11}+K_{22}\right)<0 .
$$

We must also comment on the limitation of our model caused by a limited number of terms used in the series expansion. We would consider the results of the model to be reliable when $|\Theta|$ in Eq. (5) does not exceed unity radian. The experimental values are estimated to be well within this range. One can show that for small $|\Theta|$ Eq. (6) is automatically satisfied (i.e., the energy condition is very much model dependent). Thus the root existence condition Eq. (7) describes the major physical reason for the observed stripe pattern provided that $\Theta$ is within reasonable limits.

It is likely that $\Theta \neq \Phi$. Defining $a=\Phi / \Theta$ one can obtain modified conditions Eqs. (6) and (7) as follows:

$$
\begin{gathered}
3 K_{11}\left(a^{4}+a^{2}+1\right)+17 K_{22} a^{2} \\
-K_{33}\left(3 a^{4}+10 a^{2}+3\right)<0, \\
\left(K_{11}+K_{22}\right)\left(1+a^{2}\right)<0 .
\end{gathered}
$$

Since only even degrees of $a$ exist in Eqs. (8) and (9), our qualitative conclusions remain the same.

Though the twist, splay, and the bend elastic constants in the usual nematic phase are considered to be positive, theoretically it has been considered that certain molecular properties can give rise to negative values for some of the elastic constants. For example, Dozov [9] suggested that bent-core molecules could give negative values of the bend elastic constant $K_{33}$ and produce spontaneous distortions. Being dimers with an odd number of carbons in the chain length, these materials could follow this theory as studied earlier [12]. Nevertheless, Dozov's analysis cannot be directly applied to our case, as this predicts disclination lines in the planar cells to be directed normal to the rubbing direction and also predicts domains with positive and negative optical rotations in the homeotropic cells. We have not observed these experimentally. Moreover, the bend elastic constant is not involved in the root existence condition (7). However, using a similar approach, one can suggest that for dumbbell-shaped molecules (two bulky mesogens connected by a relatively thin chain), the twist elastic constant $K_{22}$ can become negative.

Related more closely to our case, an analysis of the splay-twist escaped state can be found in [13]. However, it involves nonsymmetric (hybrid) cells which implies the influence of the divergence terms $K_{24}$ (saddle-splay) and $K_{13}$ and uses a conventional nematic LC material $\left(K_{11}, K_{22}, K_{33}>0\right)$. The reported domain periodicity is proportional to a square root of the cell thickness in contrast to the linear dependence observed in our case.
Recently Ponti et al. [14] have considered quadrupolar interactions of the elliptic molecules. They predict that with increasing the eccentricity of the interaction volume, $K_{22}$ can monotonically decrease and even change sign causing possible instabilities in the nematic orientation. This model seems favorable in the light of the above results.

It is likely that on cooling from the $N_{u}-N_{x}$ transition, either $K_{11}$ or $K_{22}$ can gradually decrease from zero towards large enough negative values to satisfy the conditions (8) and (9) and trigger the formation of the stripe pattern. However our analysis does not deny the possibility of the other elastic constant(s) to be negative in this highly unusual and interesting phase.

We summarize by concluding that the chain-linked bimesogenic dimers possess a liquid crystalline nematic phase that exhibits well-defined periodic patterns due to at least one of the elastic constants $\left(K_{11}\right.$ or $\left.K_{22}\right)$ being negative. Easily controlled self-assembly properties of such materials can have potential applications in diffraction gratings and photonics.

A. Kocot, W. H. de Jeu, J. Thoen, D. J. Photinos, and G. R. Luckhurst are thanked for valuable discussions. Work was supported by FP7 EU BIND No. 216025.

*jvij@tcd.ie

[1] M.F. Grandjean, C.R. Acad. Sci. Paris 172, 71 (1921).

[2] B. H. Soffer, J. D. Margerum, A. M. Lackner, D. Boswell, A. R. Tanguay Jr., T. C. Strand, A. A. Sawchuk, and P. Chavel, Mol. Cryst. Liq. Cryst. 70, 145 (1981).

[3] A. J. Hurd, S. Fraden, F. Lonberg, and R. B. Meyer, J. Phys. (Paris) 46, 905 (1985).

[4] R. Williams, J. Chem. Phys. 39, 384 (1963).

[5] O. D. Lavrentovich and V. M. Pergamenshchik, Phys. Rev. Lett. 73, 979 (1994).

[6] A. Sparavigna, O. D. Lavrentovich, and A. Strigazzi, Phys. Rev. E 49, 1344 (1994).

[7] J. L. Ericksen, Phys. Fluids 9, 1205 (1966).

[8] V. M. Pergamenshchik, Phys. Rev. E 61, 3936 (2000).

[9] I. Dozov, Europhys. Lett. 56, 247 (2001).

[10] A. L. Alexe-Ionescu, G. Barbero, and I. Lelidis, Phys. Rev. E 66, 061705 (2002).

[11] A. Kocot, H. Xu, S. T. MacLughadha, and J. K. Vij, Mol. Cryst. Liq. Cryst. 261, 501 (1995).

[12] P. J. Barnes, A. G. Douglass, S. K. Heeks, and G. R. Luckhurst, Liq. Cryst. 13, 603 (1993).

[13] V. M. Pergamenshchik, I. Lelidis, and V. A. Uzunova, Phys. Rev. E 77, 041703 (2008).

[14] S. Ponti, F. C. M. Freire, J. C. Dias, and L. R. Evangelista, Phys. Lett. A 372, 6521 (2008). 